\title{
l'incertitude sur les résultats d'un problème de mécanique des sols ou des roches traité par la méthode des éléments finis
}


L'INCERTITUDE SUR LES RESULTATS D'UN PROBLEME DE MECANIQUE DES SOLS OU DES ROCHES TRAITE PAR LA METHODE DES ELEMENTS FINIS

Dans cette étude, on dénombre les incertitudes qui peuvent affecter les résultats d'un problème de mécanique des sols ou des roches. Dans le cas d'un calcul mené par la méthode des éléments finis, en élasticité linéaire, une méthode est présentée qui permet d'estimer en première approximation l'incertitude sur les résultats de l'analyse. Deux exemples simples illustrent les possibilités du programme de calcul réalisé. THE UNCERTAINTY AS TO THE RESULTS
OF A PROBLEM OF SOIL MECHANICS
OR OF ROCKS TREATED BY THE METHOD
OF FINITE ELEMENTS

In this study are listed the uncertainties that can affect the results of a problem of soil mechanics or of rocks. In the case of a calculation carried out by the method of finite elements, in linear elasticity, a method is presented which makes it possible to estimate the uncertainty as to the results of the analysis in a first approximation. Two simple examples illustrate the possibilities of the programme of calculation carried out. 


\title{
l'incertitude sur les résultats d'un problème de mécanique des sols ou des roches traité par la méthode des éléments finis
}

\author{
par Bernard CAMBOU
}

\section{INTRODUCTION}

Ces dernières années, une nouvelle méthode de calcul appelée méthode des éléments finis a été développée et s'est révélée très efficace pour traiter les problèmes de champs (champs de contraintes, de déformations, de température...). Cependant, dans le domaine de la mécanique des sols ou des roches, les paramètres et données que l'on introduit dans le calcul sont souvent mal connus et les résultats de l'analyse sont alors affectés d'une certaine incertitude. Il nous a paru intéressant de développer une méthode qui permette d'apprécier en première approximation cette incertitude pour un calcul en élasticité linéaire.

\section{L'INCERTITUDE SUR LES RESULTATS D'UN PROBLEME DE MECANIQUE DES SOLS OU DES ROCHES}

Le sol ou les roches sont des milieux hétérogènes, anisotropes et discontinus qui sont habituellement définis par un certain nombre $p$ de caractéristiques que nous nommerons $\mathrm{P}_{j}(x, y, z)$ (densité, module de Young, cohésion, angle de frottement...) ( $j$ variant de 1 à $p$ ). Ces caractéristiques, du fait de l'hétérogénéité peuvent présenter en chaque point des valeurs légèrement différentes. Ces diverses valeurs ne peuvent être définies qu'en faisant une mesure en chaque point, ce qui évidemment est impossible. En tout point les $\mathrm{P}_{i}(x, y, z)$ vont donc prendre des valeurs qu'il nous est impossible de connaître avec certitude. A un point donné de l'espace $(x, y, z)$ correspondra donc une valeur simplement probable de $\mathrm{P}_{j}$ (c'est-à-dire un point de la courbe de fréquence de $\mathrm{P}_{j}$ ). $\mathrm{P}_{j}$ obéit donc à la définition d'un processus stochastique. On considèrera donc les caractéristiques du sol $\mathrm{P}_{i}$ comme des processus stochastiques spaciaux (voir Cornell [5]).

Dans le domaine de la mécanique des sols ou des roches, on peut considérer que les incertitudes qui influent sur les résultats d'une étude sont de trois types.

\subsection{L'incertitude sur la connaissance des caractéristiques du matériau}

On peut définir trois causes à cette incertitude :

1) Les caractéristiques du milieu $\left(\mathrm{P}_{j}(x, y, z)\right)$ sont des processus stochastiques dans l'espace, mais, pour limiter le nombre de paramètres dans les calculs, on est amené à considérer des valeurs moyennes spatiales (notées $\left\langle\mathrm{P}_{i}\right\rangle i$ ) sur des domaines $i$.

$$
\left\langle\mathrm{P}_{i}>i=\frac{\iiint_{\mathrm{V}_{i}} \mathrm{P}_{j}(x, y, z)}{\iiint_{\mathrm{V} i} d x d y d z}\right.
$$

L'espérance mathématique de $\left\langle\mathrm{P}_{j}\right\rangle i$, sa variance et sa covariance sont alors égales d'après Cornell [5] à :

$$
\begin{aligned}
& \mathrm{E}\left[<\mathrm{P}_{i}>i\right]=\frac{1}{\mathrm{~V}_{i}} \iiint_{\mathrm{V}_{i}}\left\{\mathrm{E}\left[\mathrm{P}_{i}\left(x_{1}, y_{1} z_{1}\right)\right]\right\} d x_{1} d y_{1} d z_{1} \\
& \operatorname{VAR}\left[<\mathrm{P}_{i}>i\right]=\frac{1}{\mathrm{~V}_{i}^{2}} \iiint_{\mathrm{V}_{i}} \iiint_{\mathrm{v} i} \operatorname{COV}\left[\mathrm{P}_{i_{1}}, \mathrm{P}_{i_{2}}\right] d x_{1} d y_{1} d z_{1} d x_{2} d y_{2} d z_{2} \\
& \operatorname{COV}\left[<\mathrm{P}_{j}>i,<\mathrm{P}_{e}>k\right]=\frac{1}{\mathrm{~V}_{i} \mathrm{~V}_{k}} \iiint_{\mathrm{v} i} \iiint_{\mathrm{v} k} \operatorname{COV}\left[\mathrm{P}_{i 1}, \mathrm{P}_{e 3}\right] d x_{1} d y_{1} d z_{1} d x_{3} d y_{3} d z_{3}
\end{aligned}
$$

$\left(x_{1}, y_{1}, z_{1}\right)$ et $\left(x_{2}, y_{2}, z_{2}\right)$ sont les coordonnées de deux points quelconques du volume $v_{j}$, où les valeurs des caractéristiques du milieu sont $\mathrm{P}_{i 1}$ et $\mathrm{P}_{i 2},\left(x_{3}, y_{3}, z_{3}\right)$ sont les coordonnées d'un point quelconque du volume $\mathrm{V}_{k}$ où les valeurs des caractéristiques sont $\mathrm{P}_{e 3}$.

$\mathrm{Si}$ les volumes considérés $\mathrm{V}_{t}$ sont suffisamment grands et homogènes, on peut admettre (Resendiz, Herrera [8]) qu'il n'existe pas de corrélations entre les propriétés des divers points du milieu. On pourra donc écrire :

$$
\operatorname{COV}\left[\mathrm{P}_{j_{1}}, \mathrm{P}_{i_{2}}\right]=0
$$

Donc, d'après les équations (1) :

$$
\begin{gathered}
\operatorname{VAR}\left[<\mathrm{P}_{i}>i\right]=0 \\
\operatorname{COV}\left[\left\langle\mathrm{P}_{i}>i,<\mathrm{P}_{e}>k\right]=0\right.
\end{gathered}
$$

Ainsi, les variables $\left\langle\mathrm{P}_{i}\right\rangle i$ sont des variables parfaitement déterminées.

Le fait d'utiliser les variables $\left\langle\mathrm{P}_{i}\right\rangle i$ à la place des $P_{j}$ n'introduit aucune incertitude supplémentaire sur les résultats de l'analyse.

2) Les valeurs moyennes spatiales des caractéristiques du milieu s'obtiennent à partir d'essais dont les résultats dépendent de divers facteurs aléatoires (opérateurs, appareils, conditions atmosphériques...). Soient $\mathrm{Q}_{j}(x, y, z)$ les propriétés observées et $\mathrm{P}_{j}(x, y, z)$ les propriétés réelles.

$$
\mathrm{Q}_{i}(x, y, z)=\mathrm{P}_{j}(x, y, z)+\varepsilon_{j}
$$

$\varepsilon_{j}$ est un terme aléatoire d'erreur ayant une valeur moyenne nulle si le résultat de l'essai n'est pas entaché d'une erreur systématique.

Les fonctions $\varepsilon_{i}$ sont indépendantes entre elles et indépendantes des $\mathrm{P}_{j}$.

On pourra donc décrire en supposant que $\mathrm{E}\left[\varepsilon_{\mathrm{j}}\right]=0$ 


$$
\begin{gathered}
\mathrm{E}\left[\mathrm{Q}_{i}\right]=\mathrm{E}\left[\mathrm{P}_{i}\right] \\
\operatorname{VAR}\left[\mathrm{Q}_{j}\right]=\mathrm{VAR}\left[\mathrm{P}_{i}\right]+\mathrm{VAR}\left[\varepsilon_{j}\right] \\
\operatorname{COV}\left[\mathrm{Q}_{i_{1}}, \mathrm{Q}_{e_{2}}\right]=\mathrm{E}\left[\mathrm{Q}_{i_{1}} \mathrm{Q}_{e_{2}}\right]-\mathrm{E}\left[\mathrm{Q}_{j_{1}}\right] \mathrm{E}\left[\mathrm{Q}_{e_{2}}\right] \\
=\mathrm{E}\left[\mathrm{P}_{j_{1}} \mathrm{P}_{e_{2}}\right]-\mathrm{E}\left[\mathrm{P}_{i_{1}}\right] \mathrm{E}\left[\mathrm{P}_{e_{2}}\right]=\operatorname{COV}\left[\mathrm{P}_{i_{1}}, \mathrm{P}_{e_{2}}\right] \\
\operatorname{COV}\left[\mathrm{Q}_{j_{1}}, \mathrm{Q}_{e_{2}}\right]=\operatorname{COV}\left[\mathrm{P}_{i_{1}}, \mathrm{P}_{e_{2}}\right]
\end{gathered}
$$

Dans $\mathrm{Q}_{i i}$ et $\mathrm{P}_{j i}$ le premier indice se rapporte au type de caractéristique et le deuxième au point où l'on considère cette caractéristique.

Soit $\rho\left(Q_{i 1}, Q_{e_{2}}\right)$ le coefficient de corrélation entre $\mathrm{Q}_{i_{1}}$ et $\mathrm{Q}_{e_{2}}$, on aura :

$$
\begin{aligned}
& \rho\left(Q_{i 1}, Q_{e_{2}}\right)=\frac{\operatorname{COV}\left[Q_{i 1}, Q_{e_{2}}\right]}{\sqrt{\operatorname{VAR}\left[Q_{i_{1}}\right] \operatorname{VAR}\left[Q_{e_{2}}\right]}} \\
& =\frac{\operatorname{COV}\left[Q_{i_{1}}, Q_{e_{2}}\right]}{\sqrt{\left(\operatorname{VAR}\left[P_{j_{1}}\right]+\operatorname{VAR}\left[\varepsilon_{i}\right]\right)\left(\operatorname{VAR}\left[P_{e_{2}}\right]+\operatorname{VAR}\left[\varepsilon_{e}\right]\right)}}
\end{aligned}
$$

$\operatorname{VAR}\left[\varepsilon_{i}\right]$ et VAR $\left[\varepsilon_{e}\right]$ étant positifs, on aura :

$$
\rho\left(\mathrm{Q}_{j_{1}}, \mathrm{Q}_{e_{2}}\right)<\rho\left(\mathrm{P}_{i_{1}}, \mathrm{P}_{e_{2}}\right)
$$

L'incertitude due au type et forme des essais va donc diminuer le degré de corrélation entre les caractéristiques ponctuelles du milieu.

Mais d'après (1) pour les moyennes spatiales, on aura :

$$
\begin{gathered}
\mathrm{E}\left[<\mathrm{Q}_{j}>i\right]=\mathrm{E}\left[<\mathrm{P}_{i}>i\right] \\
\operatorname{VAR}\left[<\mathrm{Q}_{i}>i\right]=\operatorname{VAR}\left[<\mathrm{P}_{j}>i\right]
\end{gathered}
$$

$\operatorname{COV}\left[\left\langle\mathrm{Q}_{j}\right\rangle i,\left\langle\mathrm{Q}_{e}\right\rangle k\right]=\operatorname{COV}\left[\left\langle\mathrm{P}_{j}\right\rangle i,\left\langle\mathrm{P}_{e}\right\rangle k\right]$

Donc ce type d'incertitude n'influe en aucune manière sur l'incertitude qui affecte les moyennes spatiales que l'on introduit dans le calcul.

3) Les valeurs moyennes spatiales des caractéristiques du milieu sont estimées à partir d'un nombre fini d'essais.

Pour déterminer les moyennes spatiales $\left\langle\mathrm{P}_{j}\right\rangle i$ avec certitude il faudrait connaître la valeur de $P_{i}$ en chaque point, ce qui est évidemment impossible. En fait, on calcule seulement une estimation des valeurs $\left\langle\mathrm{P}_{i}\right\rangle i$ à partir des résultats d'essais sur un certain nombre $n$ d'échantillons.

Soit $\left\langle\mathrm{P}_{i}\right\rangle i^{*}$ l'estimation de $\left\langle\mathrm{P}_{j}\right\rangle i ;\left\langle\mathrm{P}_{j}\right\rangle i^{*}$ est une variable aléatoire. Dans le cas général où la fonction $\mathrm{P}_{j}(x, y, z)$ est quelconque, il sera très difficile d'estimer les paramètres de la distribution de la variable aléatoire $\left\langle\mathrm{P}_{i}\right\rangle i^{*}$. Dans certains cas particuliers, par exemple s'il n'existe pas de corrélations dans les processus stochastiques $\mathrm{P}_{j}(x, y, z)$, on pourra utiliser une analyse de régression pour déterminer les paramètres définissant la distribution de $\left\langle\mathrm{P}_{i}\right\rangle i^{*}$.

Considérons le cas le plus simple où le processus stochastique $\mathrm{P}_{i}(x, y, z)$ admet une valeur moyenne $\mathrm{E}\left[\mathrm{P}_{i}(x, y, z)\right]$ et une variance $\operatorname{VAR}\left[\mathrm{P}_{i}(x, y, z)\right]$ indépendantes des paramètres spaciaux $(x, y, z)$ et où l'on connaît $n$ valeurs de $\mathrm{P}_{j}$. On note $\mathrm{P}_{j i}$ la valeur de $\mathrm{P}_{j}$ trouvée pour l'échantillon $i$.

On prend comme estimation de $\left\langle\mathrm{P}_{i}\right\rangle i$ la valeur

$$
\left\langle\mathrm{P}_{i}\right\rangle i^{*}=\sum_{i=1}^{n} \frac{\mathrm{P}_{j i}}{n}=m_{i}
$$

en effet, on 'a :

$\mathrm{E}\left[\left\langle\mathrm{P}_{i}\right\rangle i^{\star}\right]=\mathrm{E}\left[\sum_{i=1}^{n} \frac{\mathrm{P}_{i i}}{n}\right]=\mathrm{E}\left[\mathrm{P}_{i}\right]=\left\langle\mathrm{P}_{j}\right\rangle i$

Soit d'autre part la variable aléatoire :

$$
\begin{aligned}
& s_{i}^{2}=\frac{1}{n} \sum_{i=1}^{n}\left(\mathrm{P}_{j i}-m_{i}\right) \\
& \mathrm{E}\left[s_{i}{ }^{2}\right]=\frac{n-1}{n} \operatorname{VAR}\left[\mathrm{P}_{i}\right] \\
& \operatorname{VAR}\left[<\mathrm{P}_{i}>i^{*}\right]=\frac{\operatorname{VAR}\left[\mathrm{P}_{i}\right]}{n}=\frac{\mathrm{E}\left[s_{i}^{2}\right]}{n-1}
\end{aligned}
$$

$\mathrm{Si}$ on suppose que la variable aléatoire $\left\langle\mathrm{P}_{j}\right\rangle i^{\text {क }}$ suit une loi normale, la distribution de $\left\langle\mathrm{P}_{j}\right\rangle i^{*}$ sera donc entièrement déterminée par :

$$
\begin{aligned}
& \mathrm{E}\left[<\mathrm{P}_{i}>i^{\star}\right]=\sum_{i=1}^{n} \frac{\mathrm{P}_{j i}}{n}=m_{j} \\
& \operatorname{VAR}\left[<\mathrm{P}_{i}>i^{\star}\right]=\frac{s_{i}{ }^{2}}{n-1}
\end{aligned}
$$

\subsection{L'incertitude sur les sollicitations et conditions aux limites imposées au milieu étudié}

Ces sollicitations ou conditions aux limites peuvent être :

- Connues sans incertitude : c'est le cas souvent des conditions aux limites géométriques.

- Connues à partir d'un certain nombre d'essais (par exemple les forces tectoniques dans un massif rocheux) : les paramètres introduits pour tenir compte de ces sollicitations seront alors affectés des mêmes types d'incertitudes que les caractéristiques du sol.

- Mal connues, car difficiles à calculer dans l'état actuel de nos connaissances. On peut parfois calculer ces conditions aux limites dans certains cas simples et selon les hypothèses faites, estimer l'incertitude correspondante. Par exemple dans le cas du calcul d'une fondation, on peut considérer la répartition de contrainte dans les deux hypothèses simplificatrices d'une fondation souple et d'une fondation rigide et estimer l'incertitude découlant de l'hypothèse prise en considération.

\subsection{L'incertitude due à la méthode de calcul}

La réalité étant toujours très complexe, toute méthode de calcul implique un certain nombre d'hypothèses simplificatrices. Dans le cas d'un problème de mécanique des sols ou des roches, on considère souvent que le milieu est continu, isotrope, élastique ce qui, suivant les cas, est plus ou moins proche de la réalité.

Cette incertitude, très difficile à estimer, peut s'obtenir en comparant aux résultats expérimentaux les résultats de plusieurs calculs effectués par la même méthode sur des milieux de caractéristiques bien déterminées. 


\section{APPLICATION DE L'ANALYSE D'INCERTITUDE DU PREMIER ORDRE A LA RESOLUTION DE PROBLEMES DE MECANIQUE DES SOLS OU DES ROCHES PAR LA METHODE DES ELEMENTS FINIS EN ELASTICITE LINEAIRE}

On considère que le milieu étudié est continu, isotrope et suit une loi de comportement élastique linéaire.

La méthode des éléments finis est habituellement utilisée en mécanique des sols ou des roches pour déterminer le champ des contraintes et des déformations dans un milieu soumis à certaines sollicitations. On aboutit alors à la résolution d'un système d'équations tel que :

(2) $\{\mathrm{Y}\}=\left\{g\left(m_{1}, m_{2}, \ldots \ldots, m_{n}\right)\right\}$

$=\left[\mathrm{K}^{-1}\left(m_{1} m_{2}, \ldots \ldots, m_{n}\right)\right]\left\{\mathrm{P}\left(m_{1}, m_{2}, \ldots \ldots, m_{n}\right)\right\}$ avec

$\{\mathrm{Y}\}$ : vecteur déplacement de nœud du système,

[K] : matrice de rigidité du système,

$\{\mathrm{P}\}$ : système des forces appliquées aux nœuds,

$m_{1}, m_{2}, \ldots, m_{n}$ : valeurs moyennes spatiales des caractéristiques du milieu et des paramètres qui définissent certaines sollicitations ou conditions aux limites.

On cherche à évaluer l'incertitude qui affecte les solutions de ce système.

On applique au système (2) les résultats de l'analyse d'incertitude du premier ordre (Cornell [2])
On pourra donc raisonnablement considérer que, dans la majorité des cas, les résultats de l'analyse sont distribués normalement.

Les paramètres calculés par les équations (3) sont alors suffisants pour définir les distributions des $\mathrm{Y}_{k}$.

\subsection{Linéarité du système d'équations}

Si $g_{k}\left(m_{i}\right)$ et une fonction linéaire des $m_{i}$, les formules (3) sont parfaitement vérifiées, quelles que soient les valeurs de $\operatorname{COV}\left[m_{i}, m_{i}\right]$. Par contre, si les fonctions $g_{k}\left(m_{i}\right)$ ne sont pas linéaires, les formules (3) ne sont qu'approchées; elles ne sont alors valables que pour des valeurs de $\operatorname{COV}\left[m_{i}, m_{j}\right]$ d'autant plus petites que les fonctions de $g_{k}\left(m_{i}\right)$ sont «loin d'être linéaires".

On a donc intérêt à choisir les paramètres $m_{i}$ de telle sorte que les fonctions $g_{k}\left(m_{i}\right)$ se rapprochent le plus de la linéarité. En particulier, on aura intérêt à éviter, dans la mesure du possible, les fonctions à forte courbure (hyperbolique par exemple).

En élasticité linéaire, les fonctions $\mathrm{Y}_{k}=g_{k}\left(m_{i}\right)$ ne dépendent que des modules de Young, des coefficients de Poisson $\left(v_{i}\right)$ et des sollicitations imposées. Afin de tenir compte des sollicitations extérieures, on introduira, chaque fois que cela sera possible, des para-

$$
\left\{\begin{array}{l}
\mathrm{E}\left[\mathrm{Y}_{k}\right]=g_{k}\left(\mathrm{E}\left[m_{1}\right], \mathrm{E}\left[m_{2}\right], \ldots ., \mathrm{E}\left[m_{n}\right]\right) \\
\operatorname{VAR}\left[\mathrm{Y}_{k}\right]=\sum_{i} \sum_{j}\left(\frac{\partial g_{k}}{\partial m_{i}} / \mathrm{E}\right)\left(\frac{\partial g_{k}}{\partial m_{i}} / \mathrm{E}\right) \operatorname{COV}\left[m_{i}, m_{i}\right] \\
\operatorname{COV}\left[\mathrm{Y}_{k}, Y_{i}\right]=\sum_{i} \sum_{j}\left(\frac{\partial g_{k}}{\partial m_{i}} / \mathrm{E}\right)\left(\frac{\partial g_{t}}{\partial m_{j}} / \mathrm{E}\right) \operatorname{COV}\left[m_{i}, m_{i}\right]
\end{array}\right.
$$

où

E $\left[m_{1}\right]$ représente l'espérance mathématique de $m_{1}$; $\frac{\partial g_{k}}{\partial m_{i}} / \mathrm{E}$ représente la dérivée première de $g_{k}$ par rap$\overline{\partial m_{i}} / \mathrm{E}$ port à $m_{i}$ évaluée pour la valeur moyenne de $\mathrm{m}_{i}$.

On notera que ces formules permettent seulement de déterminer la valeur moyenne, la variance et la covariance des divers résultats de l'analyse.

Ces paramètres ne sont suffisants pour définir une distribution de probabilité que lorsque cette distribution a une forme particulière connue (distribution normale par exemple). Dans le cas général, la forme de la distribution de $\mathrm{Y}_{k}$ dépend de celle des distributions des paramètres $m_{i}$. Celles-ci peuvent avoir des formes particulières asymétriques et il semble alors difficile de connaître la forme des distributions des résultats de l'analyse. Cependant, d'après le théorème central limite, on sait que si $\mathrm{Y}_{k}$ dépend linéairement d'un grand nombre de paramètres indépendants, sa distribution est en première approximation normale. Cornell [2] a montré que la somme de $n$ paramètres ayant des distributions de forme rectangulaire (donc bien différente de la distribution normale) peut être assimilée avec une bonne précision à une distribution normale pour $n \geqslant 4$. mètres intervenant linéairement dans les fonctions $g_{k}$. Pour un sol homogène, les déformations en chaque point du milieu sont linéaires par rapport à l'inverse du module de Young et les contraintes en chaque point du milieu ne dépendent pas du module de Young. On introduira donc dans les calculs les paramètres $\mathbf{M}_{i}$, inverses des modules de Young dans chaque domaine ; le degré de linéarité de $g_{k}\left(\mathrm{M}_{i}\right)$ est alors directement lié au degré d'homogénéité du milieu. Dans la majorité des cas, les fonctions $g_{k}\left(v_{i}\right)$ étant quelconques, les paramètres $\operatorname{COV}\left[v_{i}, v_{i}\right]$ que l'on introduira dans les équations (3) devront être relativement petits.

\subsection{Incertitude sur les résultats de l'analyse due à celle sur les caractéristiques du sol}

Dans chaque domaine $i$, si on considère que les contraintes appliquées restent dans le domaine élastique, les propriétés du sol sont définies par le module de Young et le coefficient de Poisson.

Nous avons vu (paragraphe 2) que les valeurs moyennes spatiales de ces caractéristiques $(<\mathrm{M}\rangle i$, $\langle v\rangle i)$ sont estimées par des variables aléatoires $\left(\langle\mathrm{M}\rangle i^{*},\langle v\rangle i^{*}\right)$ dont on connaît la distribution (moyenne et variance).

L'incertitude sur la connaissance de $\langle\mathrm{M}\rangle i^{*}$ et $\langle v\rangle i^{*}$ entraîne une incertitude sur les résultats de l'analyse qu'on estime en utilisant les équations (3). 


\subsection{Calcul du déplacement en chaque noud} (2)

On est amené essentiellement à résoudre le système

$$
[\mathrm{K}]\{\mathrm{Y}\}=\{\mathrm{P}\}
$$

et à déterminer les dérivées $\left.\frac{\partial\{\mathrm{Y}\}}{\partial x_{i}}\right|_{\mathrm{E}}$ qui apparaissent dans les équations (3).

On notera $x_{i}$ les paramètres $\langle\mathrm{M}\rangle i^{*}$ et $\langle v\rangle i^{*}$ qui interviennent dans le système (2). En dérivant par rapport à $x_{i}$ le système (2) on obtient :

$$
\begin{aligned}
& \frac{\partial[\mathrm{K}]}{\partial x_{i}}\{\mathrm{Y}\}+[\mathrm{K}] \frac{\partial\{\mathrm{Y}\}}{\partial x_{i}}=\frac{\partial\{\mathrm{P}\}}{\partial x_{i}} \\
& {[\mathrm{~K}] \frac{\partial\{\mathrm{Y}\}}{\partial x_{i}}=\frac{\partial\{\mathrm{P}\}}{\partial x_{i}}-\frac{\partial[\mathrm{K}]}{\partial x_{i}}\{\mathrm{Y}\}}
\end{aligned}
$$

Les forces et déplacements fictifs $\left\{\mathrm{P}_{f}\right\}$ et $\left\{\mathrm{Y}_{f}\right\}$ sont définis par :

$$
\begin{aligned}
\left\{\mathrm{P}_{f}\right\} & =\frac{\partial\{\mathrm{P}\}}{\partial x_{i}}-\frac{\partial[\mathrm{K}]}{\partial x_{i}}\{\mathrm{Y}\} \\
\left\{\mathrm{Y}_{1}\right\} & =\frac{\partial\{\mathrm{Y}\}}{\partial x_{i}}
\end{aligned}
$$

et l'équation précédente s'écrit alors :

(4) $[\mathrm{K}]\left\{\mathrm{Y}_{f}\right\}=\left\{\mathrm{P}_{f}\right\}$

qui est un système identique à (2).

Ainsi donc, on résoud le système (2) par la méthode des éléments finis classique, ce qui permet d'obtenir $\{\mathrm{Y}\}$; on calcule ensuite $\frac{\partial[\mathrm{K}]}{\partial x_{i}}$ et $\frac{\partial\{\mathrm{P}\}}{\partial x_{i}}$, ce qui permet de déterminer $\left\{\mathrm{P}_{f}\right\}$.

On résoud enfin le système (4) par la méthode classique des éléments finis, ce qui permet de calculer les valeurs de $\frac{\partial\{\mathrm{Y}\}}{\partial x_{i}}$.

\subsection{Calcul des contraintes dans chaque élément}

Pour un élément donné, on a l'égalité (Zienkiewicz [9]) :

avec

$$
\{\sigma\}=[\mathrm{D}][\mathrm{B}]\{\mathrm{Y}\}
$$

$\{\sigma\}$ : tenseur des contraintes,

[D] : matrice d'élasticité.

$\{\mathrm{Y}\}$ : déplacement des nœuds de l'élément considéré,

[B] : matrice qui prend en compte la localisation de l'élément considéré.

On dérive la relation précédente par rapport à $x_{i}$ :

$$
\frac{\partial\{\sigma\}}{\partial x_{i}}=\frac{\partial[\mathrm{D}][\mathrm{B}]}{\partial x_{i}}\{\mathrm{Y}\}+[\mathrm{D}][\mathrm{B}] \frac{\partial\{\mathrm{Y}\}}{\partial x_{i}}
$$

$\frac{\partial\{\mathrm{Y}\}}{\partial x_{i}}$ est connu; on peut donc calculer $\frac{\partial\{\sigma\}}{\partial x_{i}}$.

\subsection{Incertitudes sur les résultats de l'analyse due à celle sur les sollicitations imposées au milieu.}

\subsection{Les forces de volume}

Dans la méthode des éléments finis, les forces de volume sont remplacées par des forces concentrées aux nœuds. (Les forces verticales sont habituellement dues à la pesanteur.) Dans le programme présenté, on considère que les deux systèmes de forces de volume horizontal et vertical peuvent s'écrirent :

$$
\begin{aligned}
& \left\{\mathrm{P}_{x}\right\}=a_{x}\left\{\mathrm{P}_{o x}\right\} \\
& \left\{\mathrm{P}_{y}\right\}=a_{y}\left\{\mathrm{P}_{\text {oy }}\right\}
\end{aligned}
$$

$\left\{\mathrm{P}_{o x}\right\}$ et $\left\{\mathrm{P}_{o y}\right\}$ sont des vecteurs constants parfaitement déterminés et $a_{x}$ et $a_{y}$ des variables connues avec une certaine incertitude.

On aura donc :

$$
\begin{array}{ll}
a_{x}\left\{\mathrm{P}_{o x}\right\}=\left[\mathrm{K}_{x}\right]\left\{\mathrm{Y}_{x}\right\} & \left\{\sigma_{x}\right\}=[\mathrm{D}][\mathrm{B}]\left\{\mathrm{Y}_{x}\right\} \\
a_{y}\left\{\mathrm{P}_{o y}\right\}=\left[\mathrm{K}_{y}\right]\left\{\mathrm{Y}_{y}\right\} & \left\{\sigma_{y}\right\}=[\mathrm{D}][\mathrm{B}]\left\{\mathrm{Y}_{y}\right\}
\end{array}
$$

Les vecteurs $\left\{\mathrm{Y}_{x}\right\},\left\{\mathrm{Y}_{y}\right\},\left\{\sigma_{x}\right\},\left\{\sigma_{y}\right\}$ sont linéaires par rapport à $a_{x}$ ou $a_{y}$, il sera donc facile de calculer les dérivées par rapport à $a_{x}$ et $a_{y}$ et donc l'incertitude sur les résultats de l'analyse en utilisant les équations (3).

\subsection{Les conditions aux limites}

Les conditions aux limites peuvent être de trois types :

a) Les conditions géométriques qui sont habituellement bien connues; on considère dans cette étude qu'il n'y a pas d'incertitude sur ce type de conditions.

b) Les conditions imposées pour les déplacements de certains nœuds; généralement ces déplacements imposés sont des déplacements nuls (points fixes dans le milieu) et on considère qu'il n'y a pas d'incertitude sur ces déplacements imposés.

Ceci se traduit dans le programme, en écrivant :

(5)

$$
\begin{array}{ll}
\mathrm{A}_{l l}=\mathrm{A}_{\| l} 10^{11} & \mathrm{P}_{l}=a \mathrm{~A}_{\| l} 10^{11} \\
\frac{\partial \mathrm{A}_{l l}}{\partial x_{i}}=\frac{\partial \mathrm{A}_{\| l}}{\partial x_{i}} 10^{11} & \frac{\partial \mathrm{P}_{t}}{\partial x_{i}}=\frac{\partial \mathrm{A}_{l l}}{\partial x_{i}} 10^{11} a
\end{array}
$$

avec

$a$ : déplacement imposé au nœud $l$,

$\mathrm{A}_{i j}$ : élément de la matrice de rigidité $[k]$.

On résoud le système (2) en introduisant les égalité (5), ce qui conduit à : $\mathrm{Y}_{t}=a$.

De plus :

$$
\frac{\partial\{\mathrm{P}\}}{\partial x_{i}}=\frac{\partial[k]}{\partial x_{i}}\{\mathrm{Y}\}+[\mathrm{K}] \frac{\partial\{\mathrm{Y}\}}{\partial x_{i}}
$$

donc en particulier :

$$
\begin{aligned}
\frac{\partial \mathrm{A}_{l l}}{\partial x_{i}} 10^{11} a=\frac{\partial \mathrm{A}_{l l}}{\partial x_{i}} 10^{11} \mathrm{Y}_{l} & +\mathrm{A}_{\| l} 10^{11} \frac{\partial \mathrm{Y}_{l}}{\partial x_{i}} \\
& + \text { termes négligeables }
\end{aligned}
$$

puisque : $\quad \mathrm{Y}_{l}=a \quad$ on a donc :

$$
\begin{array}{ll} 
& \mathrm{A}_{l l} 10^{11} \frac{\partial \mathrm{Y}_{l}}{\partial x_{i}}=0 \\
\text { donc : } & \frac{\partial \mathrm{Y}_{l}}{\partial x_{i}}=0
\end{array}
$$

La variance de $\mathrm{Y}_{l}$ est donc nulle, ce qui traduit bien que la position du nœud $l$ est parfaitement déterminée.

c) Les forces imposées sur les frontières.

On considère que chaque système de forces est connu avec une certaine incertitude, et qu'on peut l'écrire sous la forme :

$$
\left\{\mathrm{P}_{i}\right\}=b\left\{\mathrm{P}_{o i}\right\}
$$

où $b$ est un paramètre connu avec une certaine incertitude définie par VAR $[b]$ et $\left\{\mathrm{P}_{o i}\right\}$ un vecteur parfaitement déterminé.

Si un système de force englobe plusicurs facteurs d'incertitude, par exemple :

$$
\left\{\mathrm{P}_{i}\right\}=b_{1}\left\{\mathrm{P}_{1 j}\right\}+b_{2}\left\{\mathrm{P}_{2 j}\right\}+\ldots \ldots+b_{n}\left\{\mathrm{P}_{n i}\right\}
$$
on peut considérer chaque terme comme un système de force indépendant. 
Les déplacements et les contraintes dépendent alors linéairement des facteurs $b_{i}$, de sorte que :

$$
\frac{\partial\left\{\mathrm{Y}_{i i}\right\}}{\partial b_{i}}=\frac{\left\{\mathrm{Y}_{i i}\right\}}{b_{i}} \quad \frac{\partial\left\{\sigma_{i i}\right\}}{\partial b_{i}}=\frac{\left\{\sigma_{i i}\right\}}{b_{i}}
$$

On calcule donc facilement l'incertitude sur les résultats de l'analyse en utilisant les équations (3).

\subsection{Incertitude sur les résultats de l'analyse due à celle sur la méthode de calcul}

On considère que l'incertitude sur la méthode de calcul peut être prise en compte par un terme multiplicatif $\Delta$.

Ainsi, le système (2) peut s'écrire :

$$
\mathrm{Y}_{k}=\left[g_{k}\left(m_{1}, m_{2}, \ldots \ldots, m_{n}\right)\right] \Delta_{k}
$$

$\mathrm{Si}$ on admet que :

$$
\begin{aligned}
& \mathrm{E}\left[\Delta_{1}\right]=\mathrm{E}\left[\Delta_{2}\right]=\ldots \ldots=\mathrm{E}\left[\Delta_{m}\right]=\mathrm{E}[\Delta] \\
& \operatorname{VAR}\left[\Delta_{1}\right]=\operatorname{VAR}\left[\Delta_{2}\right]=\ldots \ddot{\operatorname{VAR}}\left[\Delta_{m}\right]=\operatorname{VAR}[\Delta] \\
& =
\end{aligned}
$$

$$
\operatorname{COV}\left[\Delta_{k}, m_{i}\right]=0
$$

On aura donc :

$$
\begin{aligned}
& \mathrm{E}\left[\mathrm{Y}_{k}\right]=\mathrm{E}\left[g_{k}\left(m_{1}, m_{2}, \ldots \ldots, m_{n}\right)\right] \mathrm{E}[\Delta] \\
& \quad \operatorname{VAR}\left[\mathrm{Y}_{k}\right]=\left(\mathrm{E}\left[g_{k}\right]\right)^{2} \operatorname{VAR}[\Delta] \\
& \quad+\operatorname{VAR}\left[g_{k}\right](\mathrm{E}[\Delta])^{2}+\operatorname{VAR}\left[g_{k}\right] \operatorname{VAR}[\Delta]
\end{aligned}
$$

$\mathrm{Si}$ on admet que le modèle mathématique ne contient pas d'erreur systématique, on a :

$$
\begin{aligned}
& \mathrm{E}[\Delta]=1 \quad \text { donc } \\
& \mathrm{E}\left[\mathrm{Y}_{k}\right]=\mathrm{E}\left[g_{k}\left(m_{1}, m_{2}, \ldots \ldots, m_{n}\right)\right] \\
& \mathrm{VAR}\left[\mathrm{Y}_{k}\right]=\left(\mathrm{E}\left[g_{k}\right]\right)^{2} \operatorname{VAR}[\Delta] \\
& \quad+\operatorname{VAR}\left[g_{k}\right](\operatorname{VAR}[\Delta]+1)
\end{aligned}
$$

La variance de $\mathrm{Y}_{k}$ ainsi déterminée caractérise donc l'incertitude globale sur les résultats de l'analyse. On prend pour variance de $g_{k}$ la somme des variances dues aux incertitudes sur les caractéristiques du milieu et aux incertitudes sur les sollicitations imposées au milieu.

\section{PROGRAMME POUR ORDINATEUR}

Le programme réalisé pour cette étude, écrit en FORTRAN IV, s'organise suivant l'organigramme donné ci-après.

\section{Entrées}

On introduit les mêmes entrées que pour un programme classique d'éléments finis (Zienkiewicz [9]) et, de plus, les variances de tous les paramètres ainsi que la covariance entre l'inverse du module de Young et le coefficient de Poisson.

\section{Sorties}

Les sorties se présentent sous forme de tables dans lesquelles on peut lire : a) pour chaque nœud les déplacements horizontaux et verticaux ainsi que les écarts types et les coefficients de variation de ces déplacements ;

b) pour chaque élément, les contraintes horizontales, verticales, les contraintes principales, l'orientation des contraintes principales, la contrainte de cisaillement maximum ainsi que leurs écarts types et leurs coefficients de variation.

On imprime une table correspondant à l'incertitude qui affecte chacun des domaines indépendants et chacun des cas de charges ainsi qu'une table où l'on considère l'incertitude globale due à toutes les incertitudes sur tous les paramètres.

\section{EXEMPLES D'APPLICATIONS}

\subsection{Premier exemple}

Cet exemple a été traité afin d'évaluer l'influence de divers paramètres sur l'incertitude qui affecte les résultats d'un problème très simple. (Pour simplifier les notations, on notera désormais $\mathrm{P}_{i j}$ les variables aléatoires $\left\langle\mathrm{P}_{j}\right\rangle i^{*}$ définies au paragraphe 2). En particulier, on a recherché l'influence de $\mathrm{E}\left[\mathrm{M}_{i}\right]$, (moyennes des inverses des modules de Young), de $\mathrm{E}\left[v_{i}\right]$ (moyennes des coefficients de Poisson), de la valeur moyenne des charges appliquées (caractérisée par un facteur multiplicatif $k$ ) ainsi que des variances $\operatorname{VAR}\left[\mathrm{M}_{i}\right]$, VAR $\left[v_{i}\right]$, VAR $\left[k_{i}\right]$ et covariance $\operatorname{COV}\left[\mathrm{M}_{i}, v_{i}\right]$.

L'exemple traité concerne un solide de longueur infinie, de section carrée soumis à une charge verticale répartie uniformément, reposant sur un substratum indéformable. Le solide est divisé en quatre domaines indépendants (fig. $1 \mathrm{~b}$ ). Les valeurs de $\mathrm{E}\left[v_{i}\right], \mathrm{E}\left[\mathrm{M}_{i}\right]$ $\operatorname{VAR}\left[v_{i}\right], \operatorname{VAR}\left[\mathrm{M}_{i}\right]$ et $\operatorname{COV}\left[\mathrm{M}_{i}, v_{i}\right]$ sont les mêmes dans chacun des domaines.

Les deux systèmes de forces appliquées cont de la forme $\left\{\mathrm{P}_{i}\right\}=k_{i}\left\{\mathrm{P}_{o}\right\},\left\{\mathrm{P}_{o}\right\}$ étant parfaitement déter- miné et $k_{i}$ étant connu avec unc certaine incortitude caractérisée par VAR $\left[k_{i}\right]$; les valeurs de $\mathrm{E}\left[k_{i}\right]$ et VAR $\left[k_{j}\right]$ sont les mêmes pour les deux systèmes de forces. Le premier système de force est composé de $F_{1}, F_{3}$, le deuxième système de force de $F_{2}$ (fig. 1).

On considère qu'il n'y a pas d'incertitude sur la méthode de calcul.

Par hypothèse, on a pris tous les coefficients de variation des $\mathrm{M}_{i}$ et $v_{i}$ égaux à une même valeur $a$. II existe de plus une certaine corrélation entre les $\mathrm{M}_{i}$ et $v_{i}$ caractérisée par un coefficient de corrélation $\rho$.

On démontre facilement (Cambou [3]) que le coefficient de variation des déplacements des nœuds et des contraintes est égal respectivement à :

$$
\left\{\begin{array}{l}
\frac{\operatorname{VAR}\left[\mathrm{Y}_{k}\right]}{\mathrm{E}\left[\mathrm{Y}_{k}\right]}=a f_{k}(\mathrm{E}[\mathrm{V}]) \\
\frac{\operatorname{VAR}\left[\sigma_{k}\right]}{\mathrm{E}\left[\sigma_{k}\right]}=a h_{k}(\mathrm{E}[\mathrm{v}])
\end{array}\right.
$$

Les coefficients de variation des déplacements et des contraintes sont donc des fonctions proportionnelles au coefficient de variation $a$ de-M, $v$ et $k$ et 


\section{ORGANIGRAMME}

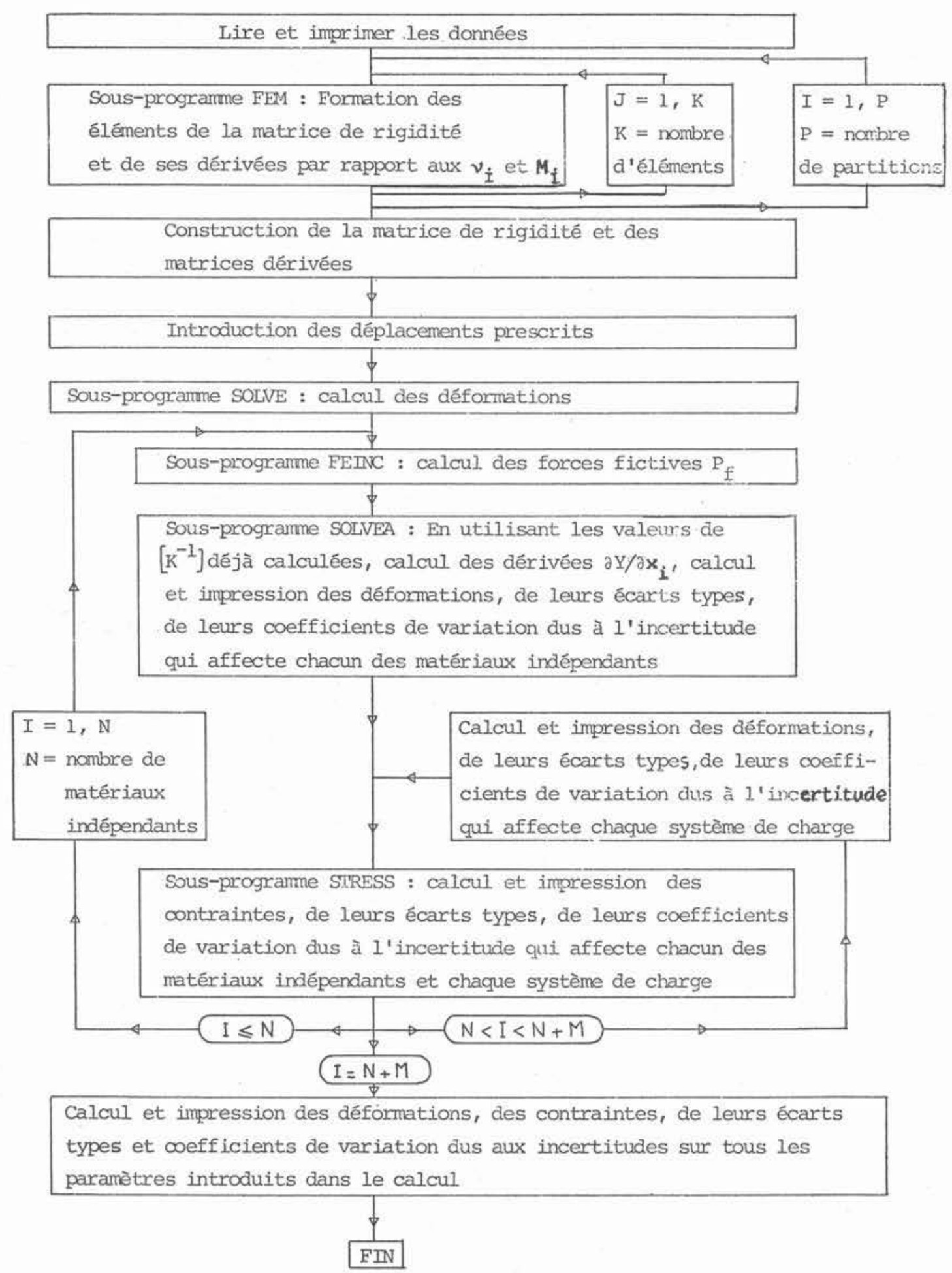


Fig. 1. - Premier exemple d'application.

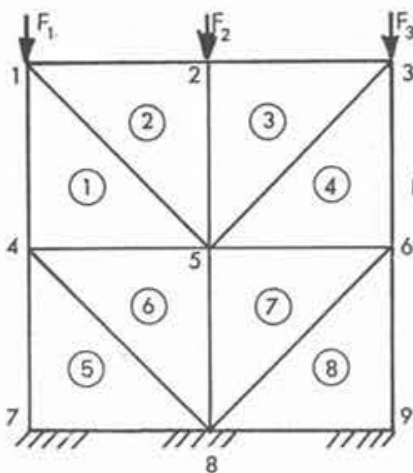

a) Maillage utilisé

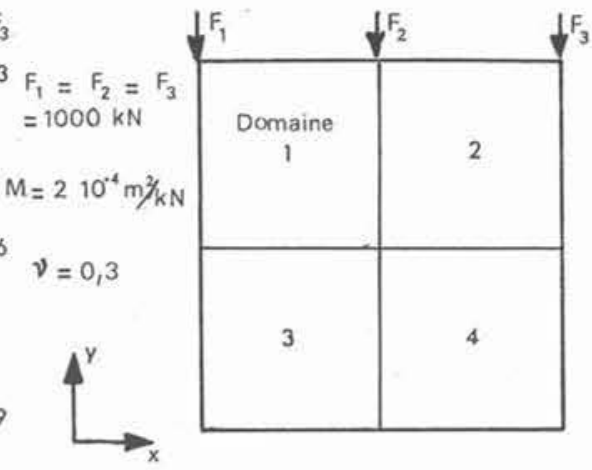

b) Localisation des quatres domaines indépendants

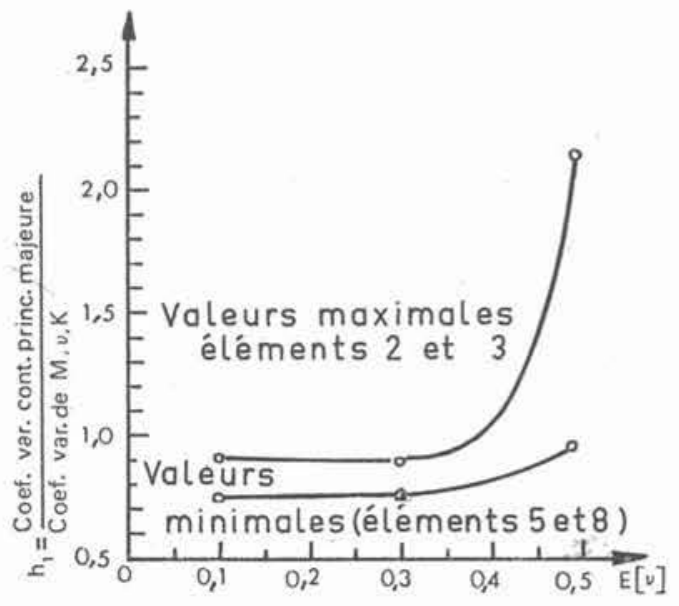

Fig. 2. - Contraintes principales majeures (premier exemple d'application).

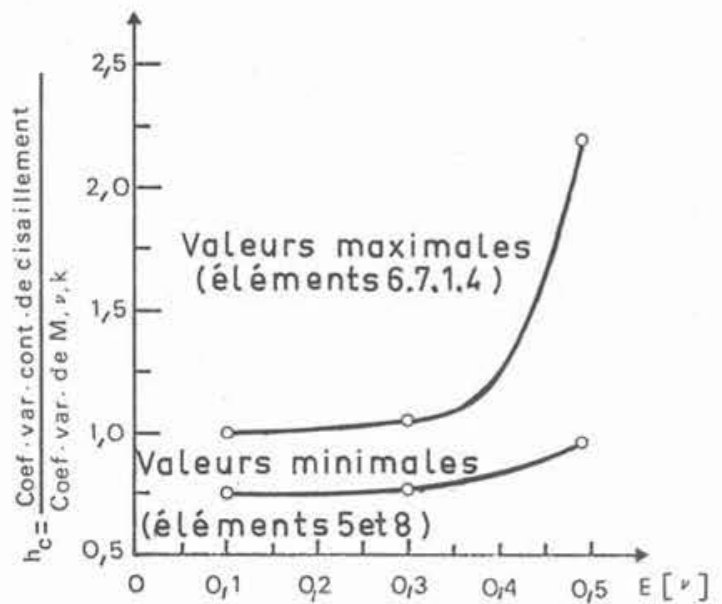

Fig. 3. - Contraintes de cisaillement (premier exemple d'application).

indépendantes de la valeur moyenne du module de Young et des forces extérieures. Ces conclusions ont été vérifiées numériquement en utilisant le programme décrit précédemment.

Les diverses fonctions $f_{k}$ et $h_{k}$ définies par (6) ont été calculées pour différentes valeurs de $\mathrm{E}[\mathrm{v}]$ $(0.1 ; 0.3$ et 0.49$)$ et sont représentées sur les figures

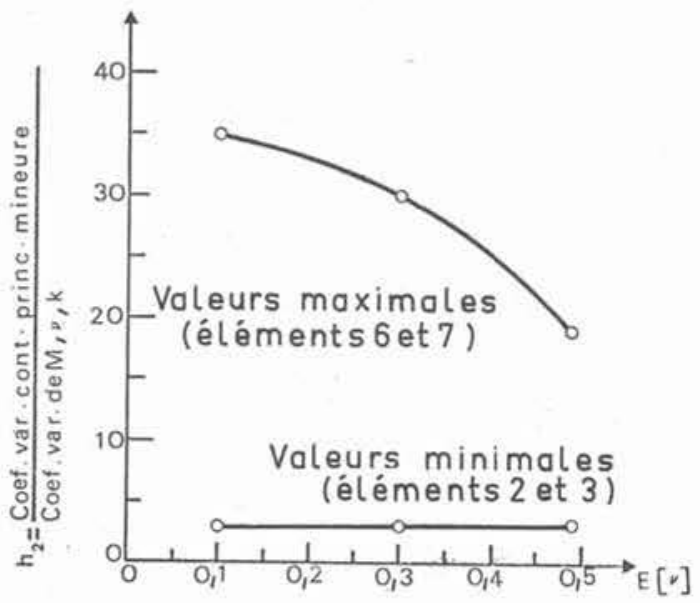

Fig. 4. - Contraintes principales mineures (premier exemple d'application.

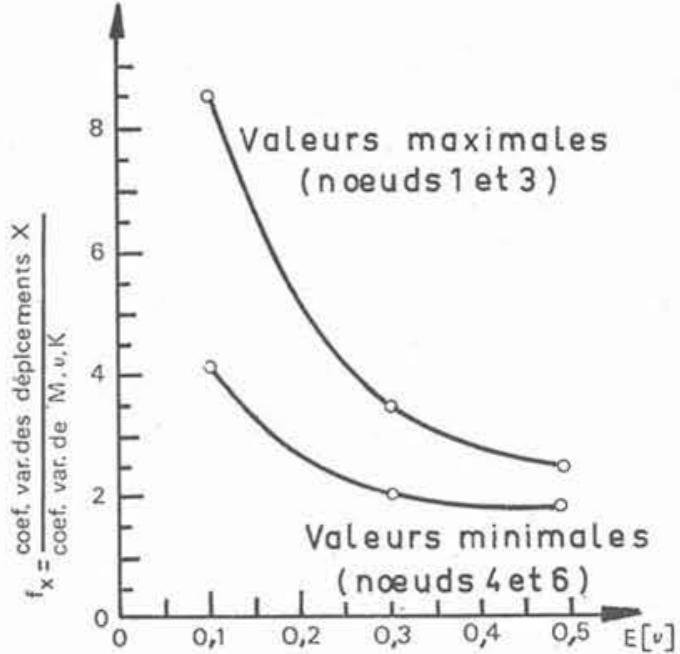

Fig. 5. - Déplacements horizontaux (premier exemple d'application).

2 à 6. On peut voir que les fonctions $f(E[v])$ et $h(\mathrm{E}[\mathrm{v}])$ dépendent de $\mathrm{E}[\mathrm{v}]$ d'une façon très sensible, en particulier les fonctions $h_{2}$ et $f_{x}$ (fig. 4 et 5); ceci met en évidence que les coefficients de variation des résultats d'une analyse par la méthode des éléments finis peuvent prendre des valeurs beaucoup plus élevées que celles des coefficients de variation des paramètres introduits dans le calcul. 


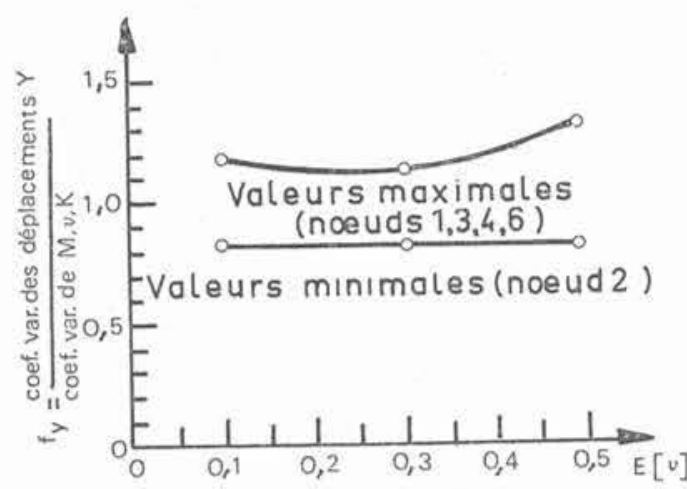

Fig. 6. - Déplacements verticaux (premier exemple d'application).

Fig. 7. - Influence de l'incertitude qui affecte chacun des paramètres sur l'incertitude qui affecte les déplacements verticaux (premier exemple d'application).

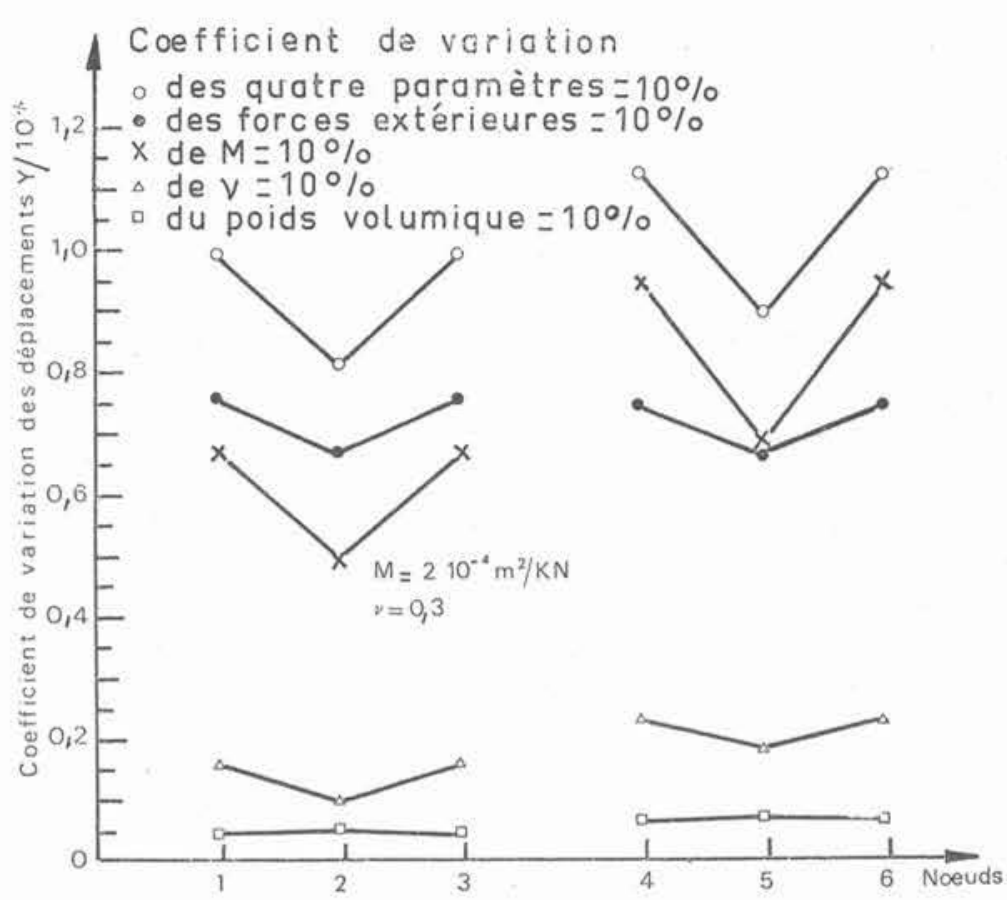

De plus, dans l'exemple traité, il semble que ce soient les incertitudes sur le module de Young et sur les forces extérieures qui influent le plus sur les incertitudes qui affectent les résultats de l'analyse ; on notera cependant que l'influence relative de chacun des paramètres introduits dans le calcul dépend de la position du point considéré (fig. 7 et 8 ).

\subsection{Second exemple}

Nous avons traité ce problème comme exemple de cas réel pour lequel il semble intéressant d'utiliser le programme décrit dans la présente étude.

Il s'agit d'analyser les déplacements élastiques à court terme et les contraintes qui se développent dans le sol au cours des excavations réalisées pour la construction du métro de Mexico. Nous avons utilisé les données du projet réalisé pour la construction de cet ouvrage (Alberro [1], Espinosa et Auvinet [6]). Les excavations s'étendaient sur plusieurs kilomètres. Dans ce cas, il est utile de définir un certain nombre de zones à peu près homogènes dans lesquelles on peut réaliser une étude statistique afin de déterminer la distribution de chaque caractéristique du sol.

Le programme permet de déterminer, en première approximation, les intervalles de confiance des déplacements et des contraintes correspondant à une certaine probabilité.

La figure 9 montre la section de l'excavation. Les caractéristiques des matériaux sont les suivantes (Espinosa et Auvinet [6], Marsal et Masari [7]) :
Béton :

$\mathrm{E}[\mathrm{M}]=10^{-7} \mathrm{~m}^{2} / \mathrm{kN} \quad$ coefficient de variation $50 \%$ $\mathrm{E}[\mathrm{v}]=0.25 \quad$ coefficient de variation $25 \%$ coefficient de corrélation entre $M$ et $\nu=0.6$

Sable : $\mathrm{E}[\mathrm{v}]=0.30 \quad$ coefficient de variation $25 \%$

Fig. 8. - Influence de l'incertitude qui affecte chacun des paramètres sur l'incertitude qui affecte les contraintes de cisaillement (premier exemple d'application).

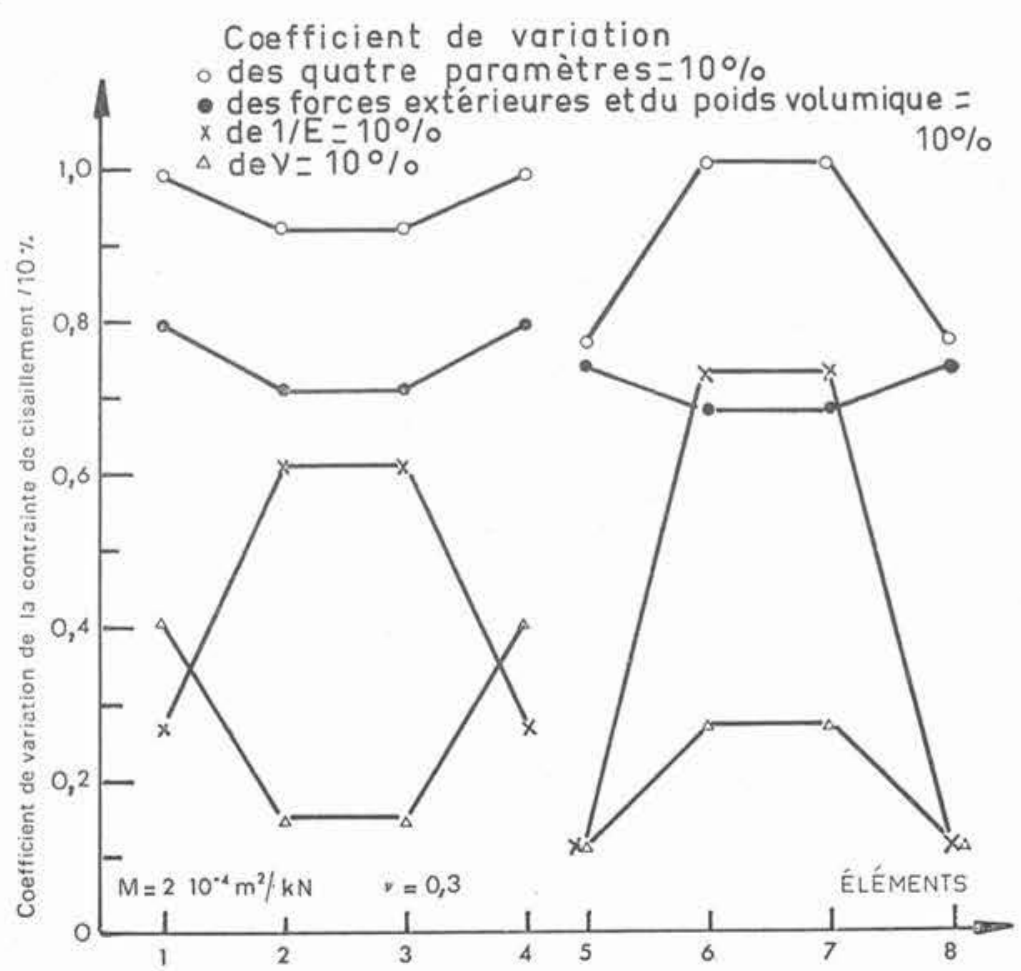

$\mathrm{E}[\mathrm{M}]=10^{-5} \mathrm{~m}^{2} / \mathrm{kN}$ coefficient de variation $30 \%$ 


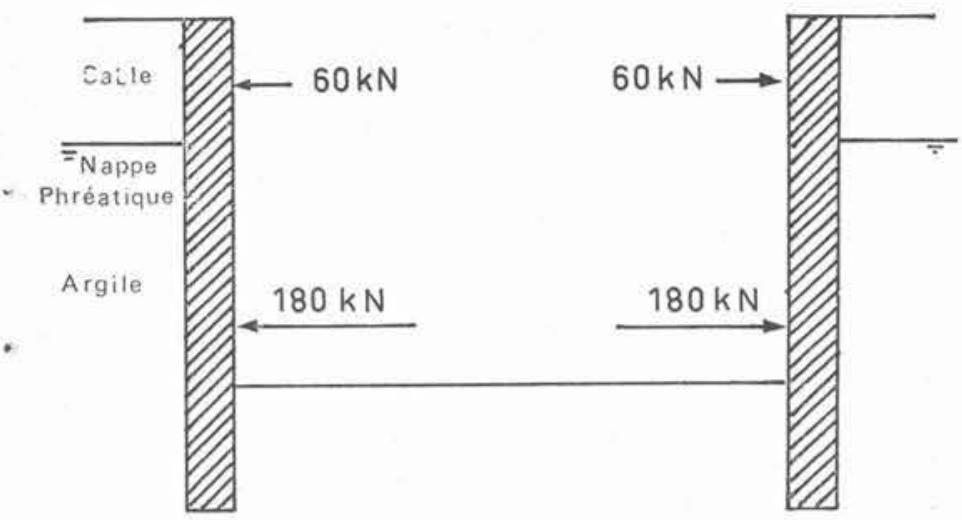

Fig. 9. - Section de l'excavation du métro de Mexico (second exemple d'application).

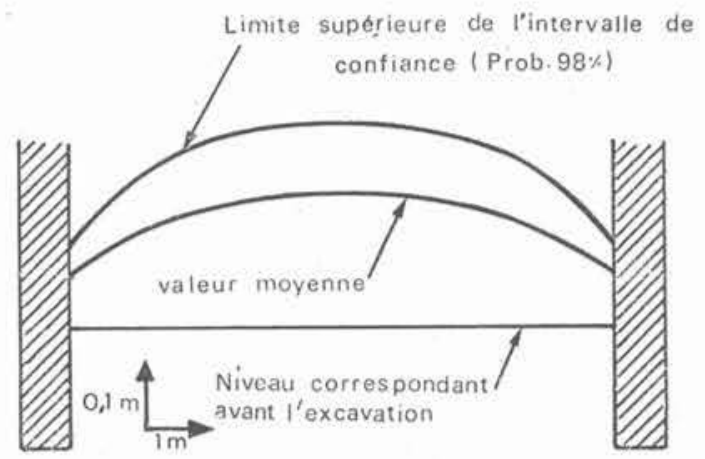

Fig. 10. - Déplacement vertical du fond de l'excavation (second exemple d'application).

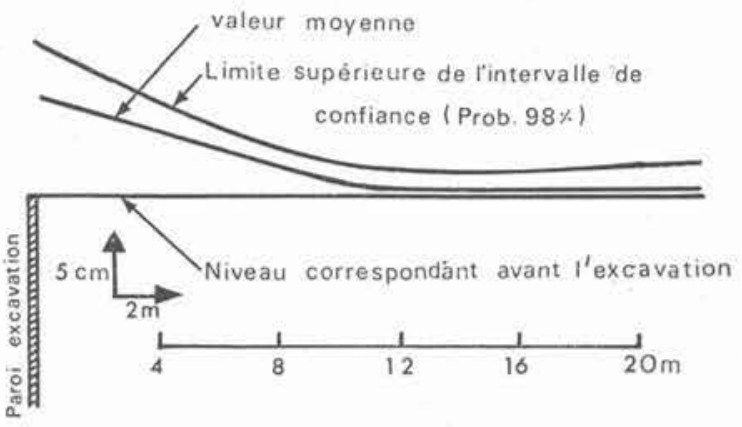

Fig. 11. - Déplacement vertical au voisinage de l'excavation (second exemple d'application).

poids volumique saturé $17.5 \mathrm{kN} / \mathrm{m}^{3}$. coefficient de corrélation entre $M$ et $v=0.6$

coefficient de corrélation entre $\mathrm{M}$ et $v=0.6$

coefficient de variation $30 \%$

coefficient de variation $25 \%$

coefficient de corrélation entre $\mathrm{M}$ et $v=0.6$

Les forces extérieures appliquées à l'excavation ont été calculées de la façon suivante.

La contrainte verticale, dirigée vers le haut qui s'applique au fond de l'excavation est égale, en module, à la contrainte verticale totale qui existait à cette profondeur avant l'excavation (on considère que l'eau est pompée hors de l'excavation).
Sur les parois de l'excavation on considère en chaque point une contrainte horizontale dirigée vers l'intérieur, égale à la contrainte totale horizontale qui s'exerçait en ce point avant la réalisation de l'excavation. On a donc :

avec

$$
\sigma_{\mathrm{HT}}=k_{o} \sigma_{\mathrm{VE}}+\sigma_{\mathrm{A}}
$$

$\sigma_{\mathrm{VE}}$ : contrainte verticale effective au point $\mathrm{P}$ avant l'excavation.

$\sigma_{\mathrm{HT}}$ : contrainte horizontale totale au point $\mathrm{P}$ après excavation.

$\sigma_{\mathrm{A}}$ : pression de l'eau au point $\mathrm{P}$.

$k_{o}$ : coefficient des terres au repos.

$k_{o}$ est un paramètre très difficile à déterminer, en particulier, il dépend de tout mouvement horizontal pendant l'excavation. On peut considérer que la valeur moyenne de $k_{o}$ est égale à 0.5 et son coefficient de variation égal à $20 \%$.

Pendant l'excavation, les parois de l'excavation étaient soutenues par deux séries de butons, qui exerçaient une force de $6 \cdot 10^{4} \mathrm{~N}$ par $\mathrm{m}$ dans la série la plus haute, et une force de $18 \cdot 10^{4} \mathrm{~N}$ par $\mathrm{m}$ dans la série basse.

On considère que seules les forces horizontales appliquées aux parois de l'excavation sont connues avec une certaine incertitude, caractérisée par VAR [ $\left.k_{0}\right]$. Les autres forces appliquées sont considérées comme parfaitement définies. On ne tient pas compte dans cette étude de l'incertitude sur la méthode de calcul utilisée.

Les figures 10 et 11 montrent la valeur moyenne et la limite supérieure de l'intervalle de confiance, correspondant à une probabilité de $98 \%$, des déplacements verticaux au fond de l'excavation et dans son voisinage. On considère que les distributions des résultats de l'analyse sont normales, ainsi donc :

$$
\mathrm{Y}_{\max }=\mathrm{E}[\mathrm{Y}]+2 \text { VAR }[\mathrm{Y}]
$$

$Y_{\max }$ : limite supérieure de l'intervalle de confiance correspondant à une probabilité de $98 \%$.

Les figures 12 et 13 montrent les courbes d'égales valeurs de l'écart type des déplacements verticaux et

\begin{tabular}{|c|c|c|c|}
\hline & $\begin{array}{l}\text { Ecart type } \\
\text { maximum }\end{array}$ & $\begin{array}{c}\text { Coefficient } \\
\text { de variation } \\
\text { correspondant }\end{array}$ & $\begin{array}{l}\text { Localisation } \\
\text { de ce maximum } \\
\text { (fig. 13) }\end{array}$ \\
\hline Déplacements verticaux $\ldots$. & $6.2 \mathrm{~cm}$ & $29 \%$ & A \\
\hline Déplacements horizontaux . & $1.2 \mathrm{~cm}$ & $50 \%$ & B \\
\hline $\begin{array}{l}\text { Contrainte principale } \\
\text { majeure } \ldots \ldots \ldots \ldots \ldots \ldots\end{array}$ & $19.3 \mathrm{kN} / \mathrm{m}^{2}$ & $23 \%$ & $\mathrm{C}$ \\
\hline $\begin{array}{l}\text { Contrainte principale } \\
\text { mineure } \ldots \ldots \ldots \ldots .\end{array}$ & $6.5 \mathrm{kN} / \mathrm{m}^{2}$ & $45 \%$ & D \\
\hline $\begin{array}{l}\text { Contrainte } \\
\text { de cisaillement ... }\end{array}$ & $10.4 \mathrm{kN} / \mathrm{m}^{2}$ & $23 \%$ & D \\
\hline
\end{tabular}
horizontaux.

On peut souligner que, en général, les coefficients de variation des déplacements horizontaux et des contraintes principales mineures sont plus élevés que les coefficients de variation des déplacements verticaux, des contraintes principales majeures et des contraintes de cisaillement. 


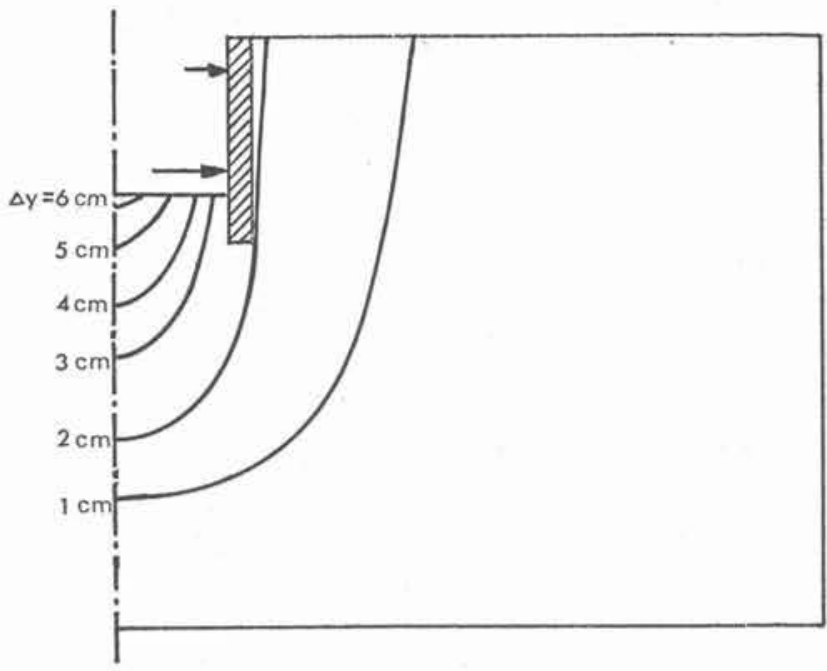

Fig. 12. - Courbes d'égales valeurs de l'écart type des déplacements verticaux $(\Delta y)$; (second exemple d'application).

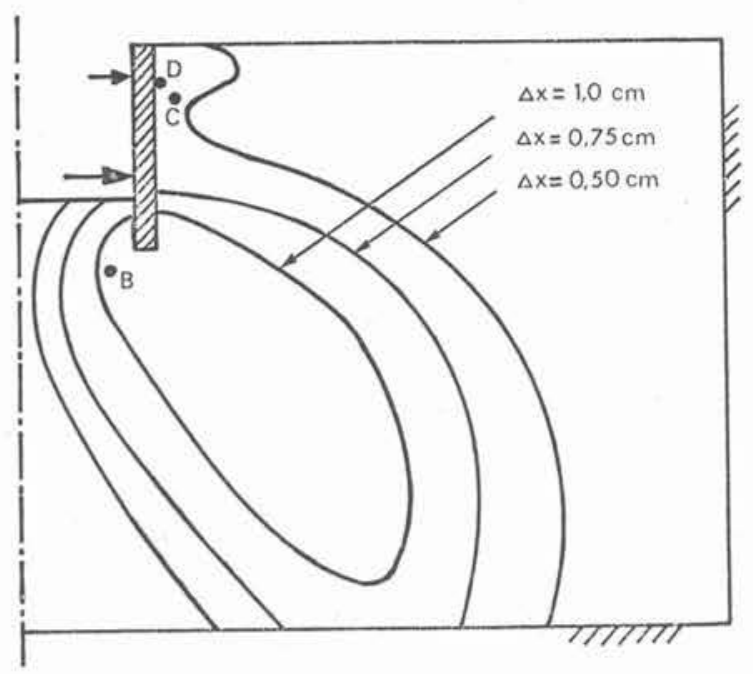

Fig. 13. - Courbes d'égales valeurs de l'écart type des déplacements horizontaux $(\Delta \mathrm{x})$; (second exemple d'application).

\section{CONCLUSIONS}

La méthode de calcul développée ici permet une première évaluation de l'incertitude sur les résultats d'une analyse par la méthode des éléments finis à partir de l'estimation des incertitudes qui affectent les divers paramètres introduits dans le calcul.

Cette méthode paraît particulièrement intéressante dans les trois cas suivants :

1) Pour tout problème qui peut être résolu par la méthode des éléments finis en élasticité linéaire, cette méthode permet d'évaluer la sensibilité des résultats de l'analyse par rapport aux divers paramètres introduits dans le calcul. En particulier, les exemples numériques montrent que cette sensibilité dépend de la valeur moyenne du coefficient de Poisson, de la localisation du point considéré, et que les coefficients de variation des résultats de l'analyse peuvent prendre des valeurs beaucoup plus élevées que ceux des paramètres introduits dans le calcul.

2) Dans le cas d'une étude par la méthode des éléments finis en élasticité linéaire pour laquelle on dispose de résultats expérimentaux, cette méthode permet de juger si les différences entre les résultats du calcul et les résultats expérimentaux peuvent être expliquées par les erreurs faites sur les données introduites dans le calcul ou si la méthode de calcul n'est pas appropriée dans le cas considéré.

3) Lorsqu'on réalise l'étude d'un ouvrage de dimensions importantes (canal, autoroute, métro) par la méthode des éléments finis en élasticité linéaire, il sera intéressant de faire une étude statistique des propriétés du sol tout au long de l'ouvrage. La méthode de calcul décrite ici peut fournir un ordre de grandeur des déplacements et des contraintes maxima de l'intervalle de confiance correspondant à une probabilité donnée.

\section{Remerciements :}

Cette étude a été réalisée pour la majeure partie à l' " Instituto de Ingenieria » de l'Université de Mexico (Mexique). Je tiens en particulier à remercier le professeur Auvinet pour son aide précieuse tout au long de cette étude.

\section{BIBLIOGRAPHIE}

[1] ALBERRO (J). - « Mediciones efectuadas en las excavaciones del metro de Mexico ». Ingenieria, Mexico (Juil.-Sept. 1970)

[2] BENJAMIN (J.R.) and CORNELL (C.A.). "Probability, statistics and decisions for civil engineers ». Mc Graw-Hill book Co, New York (1970).

[3] CAMBOU (B). - " Metodo del elemento finito, analysis de incertidumbre de primer orden " . Instituto de Ingenieria, Mexico (Jan. 1975).

[4] CORNELL (C.A.). - " Stochastic process models in structural engineering ". Dept. of civil engineering, report R 34. Stanford University, Standford Cal. (1964).

[5] CORNELL (C.A.). - " First-order uncertainty analysis of soils deformations and stability ».
" Conference on application of statistics and probability to soil and structural engineering ». HongKong (1971).

[6] ESPINOSA (L.), AUVINET (G.). - « Empuje de tierras a largo plazo sobre las estructuras tipicas del metro ». Instituto de Ingeniera, Mexico.

[7] MARSAL (R.) y MAZARI (M.). - «El subsuelo de la ciudad de Mexico ». Facultad de Ingenieria, UNAM, Mexico (1969).

[8] RESENDIZ (D.) and HERRERA (J.). - « A probabilistic formulation of settlement controled design ». Proc. VII Int. Conf. Soil. Mech. Found. Eng. Mexico 1969, vol. 2, pp. 217-225.

[9] ZIENKIEWICZ (O.C.). - «The finite element method in Engineering Science ", Mc. Graw-Hill publishing compagny (1971). 